\section{Pregnancy Outcomes in Patients with Psoriatic Arthritis}

\section{To the Editor:}

Historically, pregnancy has been considered to have a beneficial effect upon inflammatory arthritis, with around $75 \%$ of women with rheumatoid arthritis (RA) reportedly improving. These findings were mostly from retrospective studies, which lacked objective measures of disease activity ${ }^{1}$. Modern prospective studies using validated measures of disease activity reveal less impressive ameliorative effects of pregnancy on inflammatory disease activity, reporting that around 50\% of women with active RA improve during pregnancy ${ }^{2}$. Further, it is now recognized that active disease in women with inflammatory arthritis is associated with adverse pregnancy outcomes $^{3}$. Most studies, however, have focused on patients with RA and there is much less information on disease activity and pregnancy outcomes in women with psoriatic arthritis (PsA).

Therefore, we carried out a retrospective analysis of all patients with PsA followed during pregnancy in a specialized obstetric rheumatology clinic at University College London Hospital (UCLH) over a 7-year period. We specifically examined the effect of pregnancy on PsA disease activity and the effect of PsA on pregnancy outcomes. A total of 16 pregnancies in 14 consecutive patients with PsA referred to the obstetric rheumatology clinic at UCLH from 2008 to 2014 were identified. Their medical records 6 months before, during, and 4 months after pregnancy were then examined to record PsA disease activity using physician's global activity, medication history, and maternal and fetal outcomes.

Pregnancy outcomes and disease activity are shown in Table 1 and Table 2. Disease activity throughout pregnancy was available in 14 pregnancies that proceeded to term ( 2 patients had a first trimester miscarriage for which no data are available). Ten pre-pregnancy patients of the total 14 patients had mild disease activity, with 4 patients having moderate and severe disease activity. Of the 10 patients with mild disease activity, 4 had worsening disease activity during pregnancy and 4 postpartum. Those with moderate
Table 2. Disease activity outcomes in patients with psoriatic arthritis. Values are $\mathrm{n}$.

\begin{tabular}{lccc} 
Disease Activity & Pre-pregnancy & During Pregnancy & Post-pregnancy \\
\hline Mild & 10 & 6 & 4 \\
Moderate & 2 & 6 & 8 \\
Severe & 2 & 2 & 2 \\
\hline
\end{tabular}

and severe disease activity at the start of pregnancy continued to have ongoing active disease during pregnancy and postpartum. Overall, disease activity worsened through pregnancy such that $8 / 14$ patients $(57 \%)$ in both the second or third trimester and 10/14 patients $(71 \%)$ within the first 4 months of postpartum had moderate and severe disease activity compared with only 4/14 patients (29\%) pre-pregnancy. Pre-pregnancy leflunomide was stopped in 1 patient, while sulfasalazine and prednisolone were continued. Antitumor necrosis factor- $\alpha$ (anti-TNF- $\alpha$ ) therapy was continued until the end of the first trimester in all 8 patients treated with anti-TNF- $\alpha$. During pregnancy, 3 patients started receiving oral prednisone to alleviate arthritis flare. Four postpartum patients were given oral or intramuscular steroids, 1 patient recommenced methotrexate (post-breastfeeding), and 3 patients restarted anti-TNF- $\alpha$ therapy. Pregnancy outcomes were studied in 11 births at UCLH because we were unable to obtain information on the 3 other live births at different hospitals. Pregnancy outcomes included 2 first trimester miscarriages and 14 live births with no congenital malformations. The 11 live births at UCLH had mean gestation of 39.5 weeks (37-42 weeks) and mean birth weight of $3.2 \mathrm{~kg}(2.9-3.8 \mathrm{~kg})$.

Two other studies of PsA in pregnancy, published in abstract form, yielded similar findings. A study of 42 PsA pregnancies found that $58.5 \%$ of patients noted improved or low disease activity while $31.7 \%$ had worsening or ongoing high disease activity during pregnancy, and $45 \%$ had increased

Table 1. Obstetric history, pregnancy, and fetal outcomes in PsA. Values are $\mathrm{n}$ unless otherwise specified.

\begin{tabular}{lc}
\hline Variables & Values \\
\hline Diagnosis, $\mathrm{n}$ & 11 PsA \\
& 3 axSpA and psoriasis \\
Previous obstetric history, $\mathrm{n}$ & 3 miscarriages \\
& 10 live births \\
Therapy pre-pregnancy, $\mathrm{n}$ & 4 NSAID alone \\
& $3 \mathrm{SSZ} \pm$ PSL \\
& 8 anti-TNF- $\alpha$ therapy \pm SSZ \\
Therapy intervention during pregnancy, $\mathrm{n}$ & $1 \mathrm{LEF}^{2}$ \\
Therapy intervention postpartum, $\mathrm{n}$ & 3 started PSL \\
& 1 restarted MTX \\
Pregnancy outcomes & 3 given oral or intramuscular steroids \\
Median age at delivery, yrs & 3 restarted anti-TNF- $\alpha$ therapy \\
Birth outcome & 34 \\
Maternal complications & 14 live births, 2 first trimester miscarriages \\
Mode of delivery & None \\
Fetal outcomes & \\
Birth weight, kg, mean (range) & 8 vaginal; 2 elective, 1 emergency cesarean \\
Gestation, weeks, mean (range) & $3.2(2.9-3.8)$ \\
Congenital anomalies & $39.5(37-42)$ \\
\hline
\end{tabular}

${ }^{1}$ Anti-TNF- $\alpha$ was stopped during the first trimester of pregnancy in all 8 patients. ${ }^{2}$ LEF was stopped and cholestyramine washout given pre-pregnancy. ${ }^{3}$ For the 11 births at UCLH. PsA: psoriatic arthritis; NSAID: nonsteroidal antiinflammatory drug; SSZ: sulfasalazine; PSL: prednisolone; anti-TNF- $\alpha$ : antitumor necrosis factor- $\alpha$; LEF: leflunomide; UCLH: University College London Hospital; axSpA: axial spondyloarthritis; MTX: methotrexate. 
or retained high disease activity up to a 1-year postpartum period ${ }^{4}$. Another analysis of pregnancy outcomes in different forms of inflammatory arthritis reported on 8 PsA pregnancies and found that $40 \%$ of women experienced increased disease activity during pregnancy ${ }^{5}$. Findings from these studies are comparable to our own of increased PsA disease activity during and immediately post-pregnancy in an appreciable proportion of women.

Overall, we found that PsA worsens during pregnancy and postpartum. Knowledge of this increased risk of PsA disease activity in pregnancy is important during preconception counseling and advising continuation of disease-ameliorating therapies that are compatible with pregnancy.

MARIA A. MOUYIS, MBBCH, MRCP, Consultant Physician and Rheumatologist, Rheumatology Department, Northwick Park Hospital, Harrow; CATHERINE C. THORNTON, MA, BMBCh, MRCP, PhD, Consultant Rheumatologist, Rheumatology Department, Homerton Hospital, London; DAVID WILLIAMS, MB BS, PhD, FRCP, Obstetric Physician, Institute for Women's Health, University College London Hospital, London; IAN P. GILES, BSC, MBBS, PhD, FRCP, Honorary Consultant in Rheumatology, Rheumatology Department, University College London Hospital, London, UK. Address correspondence to Dr. I.P. Giles, University College London Hospital, 235 Euston Road, London, NW1 2BU, UK.

E-mail: i.giles@ucl.ac.uk

\section{REFERENCES}

1. Hazes JM, Coulie PG, Geenen V, Vermeire S, Carbonnel F, Louis E, et al. Rheumatoid arthritis and pregnancy: evolution of disease activity and pathophysiological considerations for drug use. Rheumatology 2011;50:1955-68.

2. de Man YA, Dolhain RJ, van de Geijn FE, Willemsen SP, Hazes JM. Disease activity of rheumatoid arthritis during pregnancy: results from a nationwide prospective study. Arthritis Rheum 2008;59:1241-8.

3. de Man YA, Hazes JM, van der Heide H, Willemsen SP, de Groot CJ, Steegers EA, et al. Association of higher rheumatoid arthritis disease activity during pregnancy with lower birth weight: results of a national prospective study. Arthritis Rheum 2009;60:3196-206.

4. Polachek A, Li S, Chandran V, Gladman D. Psoriatic arthritis activity during pregnancy and the postpartum period [abstract]. Arthritis Rheumatol 2015;67 Suppl 10:2520.

5. Clowse ME. Comparison of rheumatoid arthritis, ankylosing spondylitis, and psoriatic arthritis pregnancies: disease activity, treatment, and outcomes [abstract]. Arthritis Rheum 2014;66 Suppl:S706.

J Rheumatol 2017;44:1; doi:10.3899/jrheum.160929 